dere die Ionisation. Die zweite Hauptgruppe ist der elektrophysikalischen Beobachtung nach der Echomethode (Radar) gewidmet, wobei unter anderem die Beobachtung des Kopfechos, die Deutung der langdauernden Schweifechos und das Auftreten von Polarisationseffekten im reflektierten Wellenzug von Interesse sind.

Zusammenfassend kann gesagt werden, daß die Radarbeobachtung innerhalb der ersten 10 Jahre ihrer Anwendung eine Unsumme von Material erbracht hat, nicht zuletzt deshalb, weil sie unabhängig ist von Wetter und Tageslicht, und daß daraus viele neue Erkenntnisse gewonnen worden sind, auch solche, die vermeintlich gut gesicherten älteren, aus visuellen Beobachtungen gewonnenen Erkenntnissen widersprechen. Dennoch läßt sich in der Gesamtheit der Aufsätze, ohne daß dies klar ausgesprochen wird, eine gewisse Neigung zur Reaktion erkennen. Die anfängliche, wohl etwas übersteigerte Begeisterung für die neue Methode weicht einer kritischeren Auffassung, nachdem sich gezeigt hat, daß sehr exakte photographische Beobachtungen nicht unbedingt das bestätigen, was man aus den elektrophysikalischen Beobachtungen glaubte schließen zu können. Der große Wert der letzteren soll damit keinesfalls in Zweifel gezogen werden. Nur zeigt sich eben erstens, daß sie auch nicht frei von systematischen Fehlern sind, und zweitens, daß das ,Lichtmeteor" nicht unbedingt identisch zu sein braucht mit dem ,Radiometeor". Man sieht das Bestreben, kombiniert zu beobachten, d.h. gleichzeitig mit verschiedenen Methoden. Interessant ist in diesem Zusammenhang, daß die Radarmethode verminderte Empfindlichkeit gegenüber sehr raschen und sehr langsamen Meteoren zeigt, im ersten Falle, weil der Kanal ionisierter Gase im Augenblick der Entstehung durch rasche Expansion unwirksam wird, im zweiten Falle, weil dabei im Gegensatz zum Normalfall die Rekombination denselben Effekt hervorbringt.

Die Theorie der Wechselwirkung zwischen Meteorit und atmosphärischem Plasma ist qualitativ so weit entwickelt, daß wir wohl behaupten können, den Vorgang der Verdampfung des Meteoriten gut zu verstehen, besonders wenn man Autoren berücksichtigt, die in diesem Buch nicht zu Wort gekommen sind (z.B. J. HOPPE). Quantitativ aber bestehen große Widersprüche zwischen den Ansätzen verschiedenen Ursprungs, so bezüglich der Anzahl der je $\mathrm{cm}$ des Weges erzeugten freien Elektronen und der Abhängigkeit dieses Wertes von der Geschwindigkeit, welche Beziehungen auch für die Theorie der Radioechos von besonderer Bedeutung sind.

Im ganzen bestätigt das Buch, daß auf dem Gebiete der Erforschung der Meteore in den letzten 10 Jahren außerordentliche Fortschritte gemacht worden sind, die nicht allein auf der Erfindung neuer Methoden beruhen, sondern auch auf dem verstärkten Interesse der physikalischen Wissenschaften für alle Vorgänge in der hohen Atmosphäre.

\section{Hoffmerster (Sonneberg)}

Winkler, Anneliese: Die Bakterienzelle. Ein Überblick über den gegenwärtigen Stand unseres Wissens. Stuttgart: Gustav Fischer 1956. 124 S. u. 10 Tafeln. DM 11.50.

Dieses vorzïgliche Buch gibt dem Leser (sei er ein Student oder ein Fachkollege) einen Überblick über unsere gegenwärtigen Kenntnisse auf dem Gebiet der Bakterienzytologie oder Anatomie der Bakterienzelle. Die Darstellung ist objektiv, aber glücklicherweise nicht zu sehr, so daß wir auch die Ansichten der Verf. erfahren. Es ist bekannt, daß die grampositive Zellwand der Bakterien außer dem Gramkomplex (Magnesiumribonucleat plus Trägerprotein) und den Polysacchariden noch einen wechselnden Gehalt von Lipoiden und Desoxyribonucleoproteiden besitzt. Im Gegensatz hierzu stehen unsere sehr geringen Kenntnisse des morphologischen Aufbaus der gramnegativen Zellwand, die vor allem durch ihr immunchemisches Verhalten charakterisiert ist. Im Kapitel über die Kapseln der Bakterien werden wir neben anderem mit neuen Forschungsresultaten bekannt gemacht. Obwohl über den morphologischen Aufbau und die Bewegungsart der Geißeln sehr wenig bekannt ist, wird - so hören wir - heute angenommen, daß sie im Zytoplasma entspringen und da $\beta$ sie wahrscheinlich ein Basalkorn besitzen. Es ist interessant, daß nach neuen Untersuchungen auch die Spirochaeten geißelartige Fortsätze haben sollen. Das wichtige Kapitel über die bakteriellen Zellkernäquivalente oder Nucleoide wird in zwei Abschnitten behandelt: 1. Das Aussehen der Nucleoide, 2. Das Verhalten der Nucleoide. WrNKLER betont, daß wir am Vorhandensein und an der Lagerung der Nucleoide, wie sie in der Literatur beschrieben sind, nicht zweifeln können. Hingegen ist das Verhalten der Nucleoide während der Zellteilung der Bakterien von den verschiedenen Autoren in widersprechender Weise beschrieben worden. Nach Ansicht der Verf. hängt diese Uneinheitlichkeit weitgehend von der Technik der Präparation ab, wobei die Wahl des Fixierungsmittels eine ganz besonders große Rolle spielen dürfte. Der Nachweis der Nucleoide in der lebenden Zelle ist natürlich , das erstrebenswerte Ziel". Aber weder das Phasenmikroskop noch die elektronenoptische Darstellung können bis jetzt diese Forderung erfüllen. Jedenfalls liegen die Nucleoide - nach WINKLER - innerhalb der im Phasenkontrast heller erscheinenden Zonen, und nach elektronenoptischen Bildern von ultradünnen Bakterienschnitten können wir vermuten, daß die Nucleoide die durchsichtigsten und auch flüssigsten Bestandteile des Plasmas sind. Obwohl genetische Rekombinationen in Bakterien vorkommen, scheint die Übertragung einer erblichen Eigenschaft nicht durch eine sichtbare Verschmelzung von Zellen oder Kernstrukturen zu erfolgen. WrNKLER zieht in Betracht, daß die Bakterien möglicherweise bei der Vermehrung ihrer Erbsubstanz wie bei der Rekombination eigenen Gesetzen folgen. Das Kapitel über das $Z$ ytoplasma und die zytoplasmatischen Einschlïsse beschäftigt sich insbesondere mit den metachromatischen Körnchen, die mit Sicherheit Metaphosphate enthalten. Es scheint nicht berechtigt, sie mit den Mitochondrien höherer Zellen zu identifizieren. Im Kapitel über die Spore werden diejenigen Theorien besprochen, die mit den physiologischen Beobachtungen in gutem Einklang stehen. Ein Teil der Nucleoide der Mutterzelle wird in die Vorspore aufgenommen und gewinnt ein kompałteres Aussehen, während restliches Kernmaterial und Mutterzelle zugrunde gehen. Das physiologische Verhalten der ruhenden Spore, ihre Hitzeresistenz, ihre überaus lange Lebensdauer ist uns noch immer ein Rätsel. Die vorvegetativ veranderte Spove wird als ein Stadium definiert, das zwischen dem Verlust der Lichtbrechung und dem Auskeimen der Spore liegt. Das Kapitel über abwegige Bakterienformen und die $L$-Phase wird eingeleitet durch eine historische Übersicht über dieses Gebiet. Die klare Abgrenzung der , abwegigen" Formen von den "L-Phasen" der Bakterien muß besonders hervorgehoben werden: ,Die abwegigen bakteriellen Formen sind zwar der Ausdruck eines echten Wachstums, das aber auf einen äußeren Reiz hin nicht mehr in den gewohnten Bahnen ablaufen kann". In dem Kapitel über die L-Phase der Bakterien vermeidet WinkLER die Verwirrung, die in vielen Arbeiten auf diesem Gebiet gefunden wird, und es ist verdienstvoll, daß sie bei Mitteilungen über gelungene Umwandlungsversuche zut scharfen Unterscheidung auffordert ,zwischen denjenigen, die über eine weitere Züchtung der Organismen in mehreren Passagen als $L$-Formen und denen, die nur über eine reversible Umwandlung berichten". WINkLER betont mit Recht die Schwierigkeiten der Untersuchungen und die Täuschungen, denen die Untersucher zum Opfer fallen können. Unter anderem werden die Theorien besprochen, die in der $L$-Phase einen Entwicklungszyklus sehen. Die Ref. möchte Winkiers Auffassung unterstreichen, daß nämlich die $L$-Phase der sichtbare Ausdruck für eine weitgehende Adaptation an veränderte Lebensbedingungen sei und daß hier vor allem das Stoffwechselgeschehen in der weiteren Forschung berücksichtigt werden sollte. Am Schluß werden Ratschläge über die einschlägigen Untersuchungsmethoden gegeben.

Das Buch kann wegen seiner flüssigen Darstellung, seiner kxritischen Sichtung des Materials, der umfassenden Literaturangaben und schließlich der wertvollen persönlichen Gesichtspunkte und Hinweise von Professor WINKLER aufs wärmste empfohlen werden. E. KLIENEBERGER-NOBEL (London)

\title{
BERICHTIGUNG
}

zu der Kurzen Originalmitteilung ,,Die Abkürzung der Samenruhe bei Arabidopsis durch Gibberellinsäure“ von F. J. KRIBBEN [Naturwiss. 44, 313 (1957)]. In Zeile $15 \mathrm{muB}$ es heißen: scariola (statt sativa) und in Zeile 24: 0,01\% (statt $0,0001 \%$ ). Herausgeber und verantwortlicher Redaktetr: Professor Dr. Ernst Lamla, Göttingen. - Verantwortlich für den Anzeigenteil: Günter Holtz, Berlin W 35 ,
Reichpietschufer 20. - Springer-Verlag, Berlin-Göttingen-Heidelberg. - Druck der Universitätsdruckerei H. Stürtz AG., Würzburg. - Printed in Germany 